\title{
Study of Personalized Product Design based on User Intention
}

\author{
1. Sun Wen 2. Zhang Li \\ Department Of Art and Design, GuangDong University Of Science \&Technology \\ Nancheng District, Dongguan, Guangdong
}

\begin{abstract}
After entering the 21st century, the competition in the product market has further intensified, and the demand for personalized products of users has become increasingly strong, which has led to the emergence of this new customized production model. Based on the special needs of users, the adjustment of production process and product structure, the application of advanced information technology, production technology and management technology, this paper combines with mass production and high efficiency to complete the production of customized products to achieve effective combination of product customization and mass production. From the point of view of production products, we can see that the development of new models is driven by the market. In order to survive in the fierce market competition, we must adapt to the market and meet the needs of users.
\end{abstract}

Keywords-Personalized Product Design; User Intention; Product Modern

\section{INTRODUCTION}

The material function can not fully satisfy the user's pursuit of the product, and the user pays more and more attention to the spiritual enjoyment, and this spiritual enjoyment mainly comes from the user's understanding of the product semantics. However, the perception of product semantics is a very complicated psychological cognitive process, often a succinct and non-quantitative description of some words. One of the important tasks of industrial designers in product design is to accurately grasp the user's understanding of product semantics. In the engineering design of the product, the design engineer mainly considers some quantitative technical indicators, which causes the "information asymmetry" between the nonquantitative perceptual semantic information of the industrial designer and the quantitative technical indicators of the design engineer. ", which affects the user's satisfaction with the products developed. From this point of view, the quantitative problem of perceptual semantics has become an indispensable and urgent problem to be solved in the product design process. The design practice of personalized products also needs a scientific method based on the quantitative linguistic semantics as a guide. Taking the quantitative relationship between user characteristics, user image and product modeling elements as the basis of modeling design, the traditional design method can be changed to avoid repetitive work, and the designed products are more in line with the individual requirements of users. The research of this topic is based on this consideration.

\section{PERSONALIZED PRODUCT DESIGN AND USERS` NEEDS}

At the beginning of the 20th century, Ford of the United States created a large-scale production model marked by assembly lines. Its low-cost, high-efficiency manufacturingleading production-type advantage meets users' needs in a timely manner in the short-term economic era. need. However, along with the continuous development of technology, the market operation mode has undergone great changes. Not only the advancement of science and technology and the improvement of enterprise productivity make the market no longer suffer from product shortage and oversupply, consumers' choices are getting bigger and bigger; and because of the improvement level of people's consumption, users are increasingly pursuing new and individualized, The level of demand for products is higher, and the existing development has become a situation of short supply. The standard product model of large-scale production and unified market production is affected by the market saturation and diversified demand. Without the previous sturdiness, high efficiency and market control advantages, it has promoted the new production model of product customization. Born. Modern product customization, the company wants to gain more revenue, first win the user's order, and then use the group technology, standardization technology and modular technology to design, and then customize the production method through large rules. It can be seen that in order to win the market, we must respond quickly to the needs of users and quickly design and develop products with good user satisfaction. In this case, the personalized product design is generated, and its core is the analysis and research of the user's individual needs and its grasp in product design. The user requirement is the target event of the target user in a specific scenario. User needs include both physical and spiritual aspects. The material aspect includes the material, shape, color and size of the product. These elements are quantifiable and can be quantitatively described. Compared with the designer, it is often better to grasp, and the mentally non-quantitative and confusing words are more difficult to grasp. of. Therefore, the focus of this article is on the semantic aspects of the language, including the extraction, analysis and production of questionnaires.

User imagery is an important factor influencing the user's purchase of products and spiritual enjoyment. Whether a product can attract users often leads directly to the user to purchase the product; if the user uses a product to feel comfortable or convenient, or feel good, comfortable, or simple 
to operate, high efficiency, etc., it will give users a spiritual enjoyment. The product has received favorable comments, which promoted product sales and improved the brand effect of the company. Therefore, in product design, the designer's acquisition and accurate grasp of user perception information is the key to determine the success of product design. The user's image information about the product can be obtained by the user or obtained through market research. The supply by the user does not necessarily mean that the user actively supplies related information, and the experts can analyze and summarize the products through the experts in these aspects. The testee must fill in the questionnaire designed by the designer in advance, and requires the intervention and cooperation of the tester. Shows the tester's experience. On the one hand, the information on the first-hand information, on the other hand, the information provided by the tester, all require information value and accuracy, so the product research summary is a very important link. Due to the ambiguity and non-quantitativeness of the product's perceptual information, this kind of implicit information should be presented through explicit means in the previous research to facilitate the information acquisition and post-processing analysis.

Now when consumers buy products themselves, many of them decide whether to buy this product based on their own subjective feelings. The purpose of personalized design meets the user's living habits, so that the product not only satisfies the user's physiological characteristics, but also satisfies their psychological needs, makes life more convenient and comfortable, and is more enjoyable in the process of using the product. If the emotional needs of a person resonate with the characteristics of a certain product at the sensory level, then this product is very likely to be favored by consumers. The user's emotional needs are intuitive, abstract, latent and symbolic.

In the preliminary preparation work, six products of the most consumer groups were selected in more than 300 commonly used products in daily life, laying the foundation for personalized product design. Taking humidifier products as an example, there are various forms in the market for collecting products, and more than 200 word pairs have been found for them. The methods for collecting image words mainly include brainstorming, humidifier product introduction book, magazine, network, imagery. Contact law and supermarket salesperson communication and related user image research. In more than 200 pairs of words, the words with similar levels of meaning are selected firstly, and the sensuous words with similar meanings or little contact are selected, and 40 words are finally obtained; 40 pairs of style words are divided into 20 pairs of corresponding words. The semantic phrase, find 30 designers with professional background, ask each person to select 8 pairs of the best description of each product modeling factor, use the relevant software to sort the questionnaire, select the most selected lyrics, and The results analyze the correlation between user characteristics, user image and product modeling elements, and finally get 8 sets of representative vocabulary pairs as follows: soft - hard, dynamic - static, cute - bored, personalized - Popularity, quality - rough, natural - artificial, modern - retro, fresh - dull, produced into a questionnaire, laying the foundation for personalized product design research.

\section{FACTORS OF PERSONALIZED PRODUCT DESIGN}

The core of personalized product design is user needs, and different users have different needs for the same product, and the understanding of product semantics will be different. In the design of personalized products, designers need to analyze their perception of products according to different users, and use reasonable styling elements to complete product design. This chapter takes the humidifier product as an example, and divides the relevant factors of product design into three different domains: product modeling element, user feature and user image. The idea of set theory is used to analyze the relationship between elements in each domain.

The main modeling elements of humidifier products can be divided into four categories: shape, color, switch and vent. The shapes include square, rectangular, cylindrical and irregular shapes; the colors include pink, blue, black and white, green and silver; the switch mainly includes touch screen, cylindrical, convex rotation, roller type, rectangular and pointed ladder; The pores mainly include a four-sided type, a round hole upward, a double hole rotation, a round hole side spray, a double up hole and a cute decorative type.

The user characteristics of users are mainly divided into seven categories: gender, age, major, education, income, region and ethnicity. The gender includes males and females; the ages are mainly divided into five ages: 0-18, 18-28, 28-40, 40-58 and 58; the occupation includes technicians, workers, teachers, students. , freelancers, staff, sales staff, business managers and farmers; qualifications include junior high school, secondary and secondary, junior college, undergraduate and graduate students; income is divided into 1500 or less, 1500-2500, 25004000, 4000-6000 And more than 6,000 yuan; the region is divided into the North, the South and Central China.

The image of the user of the humidifier product is mainly divided into eight groups of semantic meanings, which are soft and soft - hard; dynamic - static; cute - bored; personalized popular; quality - poor; Natural - artificial; modern - traditional fresh - dull. The user is a 18-28 year old designer with a male gender, graduated from a college with a monthly salary of over 6,000 , and a Han youth living in the south; the user image of the humidifier tends to be personalized, quality, modern, Fresh, hard, static, unlovable, artificial style, in terms of modeling elements, tends to irregular shape, silver color, touch screen switch and air outlet on the side of the hole. For each type of user, a hierarchical relationship diagram between the three types of elements in the form of 5 can be made. Through the diagram, the user characteristics, user image and product modeling elements are linked to complete the qualitative analysis of the relationship between the elements in the three different domains of user features, user images and product modeling elements.

When designing a personalized product, the designer needs to consider three aspects, namely user characteristics, user image and product modeling elements. Designers need to design products that are satisfactory to them based on specific user characteristics, or the key to personalized design of products is to find out the specific product styling elements that users like. From the survey of product semantics in the previous chapter, it can be seen that the differences in user 
characteristics will have different semantic understanding and understanding of the product, so it can be considered that the user feature domain is closely related to the user image domain; in terms of modeling design theory, It is generally believed that the modeling elements of the product, such as shape, color, texture, etc., are the main factors that form the user's image of the product, that is to say, the modeling element domain and the user image domain also have a close relationship. However, the relationship between the user feature domain and the product modeling element domain is not straightforward and obvious. The purpose of this paper is to infer the relationship between the user feature domain and the modeling element domain based on the relationship between the user feature domain and the user image domain, the modeling element domain and the user image domain, so as to effectively match the elements of the three domains in the design. To meet the needs of personalized product design.

For a specific user group, the user feature has only 7 elements, and its matrix form can be simplified to a row vector or a column vector. For a specific product, the modeling element first considers four major elements E1 - E4, so that the modeling element domain It can also be simplified to a row or column vector to simplify the calculation process. According to this simplified form, the importance of the four types of modeling elements that need to be considered for the specific user is calculated, and then the importance of different modeling elements in each major category is further refined, thereby designing a personalized design for the specific user. The importance of the specific styling elements that the product needs to consider, and provide guidance for individual product design.

\section{PERSONALIZED PRODUCT DESIGN PROCESS AND SOFTWARE OVERALL ARCHITECTURE DESIGN}

The first part is the design part. Firstly, the input of user characteristics is mapped according to the calculation method of the previous theoretical research, and the design guidance is obtained. Then the program library is selected. If the design program library reaches the user satisfaction, the result can be directly output, if the user is not satisfied. The existing design scheme enters the prototype solution library, selects the satisfactory prototype and directly links the 3D Rhinoceros software for detailed design. After the design is completed, the new product is evaluated in terms of semantic meaning, and the final information is stored in the data to expand the database. Resources. The second part is mainly the retrieval part, the input of the style language vocabulary, directly linked to the database for retrieval, and then according to the user characteristics and style semantic meaning mapping calculation, get the design guidance, and then enter the selection of the program library, followed by the first part The method of operation is the same. This paper mainly studies the mapping calculation part. The database is mainly divided into user feature database, style semantic library, modeling element library, design solution library and prototype solution library. The user feature library and style language element library are mainly used to retrieve and For mapping calculation, the design solution library and the prototype solution library mainly provide design convenience to the designer after design guidance, and help the designer to improve efficiency when designing the product.

The rise of $3 \mathrm{D}$ building technology is based on the development of computer technology. The virtual model simulated by computer technology can be presented to the user through the computer monitor like a real object. The main methods of virtual 3D building in the field of computer-aided design are divided into Three categories: wireframe construction, surface construction and physical construction. The wire frame type is mainly used in the field of twodimensional computer-aided design. It has the advantages of convenient manipulation, small storage capacity and convenient modification, but the ability to reflect the threedimensional spatial relationship is slightly insufficient. The surface construction can easily establish precision and tension. In the complex surface modeling, in the 3D building software, complex surfaces are mostly realized by surface modeling. The entity constructs a three-dimensional triangular or quadrilateral mesh data. The entity model is based on the data points of the plane polygon, that is, the vertices of the polygon. It is composed of a number of lines running through the space of the polygon and in different planes. The interior of the model is an entity composed of lines. Therefore, the construction method of the entity model is the basic method of establishing a virtual three-dimensional model.

Access is more powerful than Excel in dealing with large amounts of data, but using Access to complete data processing tasks is more complicated than Excel. The result of this complexity is that it is more powerful. Access is organized and there is a strict correlation between the various objects. Because of the rationality and relevance, it is the foundation of Access's powerful data processing capabilities. Therefore, in operation, we must abide by the software's normative rules, and the method of Access processing data can be regarded as a company's organizational structure. Access is easy to use, and it can be used more sensitively and quickly in the desktop database system. It can also be further developed on its own desktop database application (UI), and can also be used for front-end development tools and other database development. Applications (such as SQL Server, DB2, Oracle, etc.) are commonly used by developers and data analysts of the software to complete development tasks, and developers who do not use the software often can use it to complete work tasks, as opposed to others. Software is better to master, in summary, Access is more suitable for developing system software.

The type element library is mainly used for retrieving and mapping calculations. The design solution library and the prototype solution library mainly provide design convenience to the designer after design guidance, and help the designer to improve efficiency when designing the product.

The type element library is mainly used for retrieving and mapping calculations. The design solution library and the prototype solution library mainly provide design convenience to the designer after design guidance, and help the designer to improve efficiency when designing the product. The type element library is mainly used for retrieving and mapping calculations. The design solution library and the prototype solution library mainly provide design convenience to the 
designer after design guidance, and help the designer to improve efficiency when designing the product.

\section{TECHNICAL IMPLEMENTATION OF SOFTWARE SYSTEM}

A database is a collection of data consisting of one or more sheets used to store the corresponding data. In order to realize the callability of the software system, this paper uses the database technology to store and manage the corresponding, which can help the designer to design the product, and use the relevant factor analysis database to display, find and call. Not only can the method of personalized product design be more accurate, but the method can be more convenient and faster. Microsoft Office Access effectively combines the development tools of database software with the graphical interface of the user, and is also a database management system. Access mainly consists of seven objects: table, lookup, macro, module form, report, and data page. Used to collect, store, and process different types of information. Database creation and reading can be done by using the appropriate action buttons, or by using SQL as the language for database deconstruction lookups. In addition, Microsoft Database provides a set of database access standards for open database connections and open data connections. The database is suitable for analyzing and organizing data and classifying users' filling. It is the most basic part of design software. The most complicated and timeconsuming phase is to support the subsequent operations. This part is the basis for the design of the database. It involves the design of other stages. It needs to find all the objects to be dealt with, and then collect user requirements, basic data and data flow according to the overall design goals. The needs analysis includes three stages: information collection, classification and evaluation. Through the analysis of the system function framework, the main data of the system database are: user feature library, style semantic library, product modeling element library, design solution library, prototype solution library and so on. All the first-hand data in the database design is designed and input by the entity-contact method (E-R), which can achieve the aggregation of all data. Since the RDBMS (Relational Database Management System) is a twodimensional table as the basic management unit, the ER model ultimately describes the relationship between the tables by the two-dimensional table, and the conceptual model of the E-R model to the database is mapped to the data table conversion process. Entities in the system can be decomposed through conceptual E-R diagrams.

The operation of the database is divided into two parts: the call and the inbound. The main purpose of the call is to let the data in the database participate in the calculation, and the design guidance is obtained through the calculation of the mapping relationship; another purpose is to retrieve the content in the existing database. Is there a design that meets the needs of the user? The warehousing part mainly includes the storage of user characteristics, the storage of semantic linguistics and the design of the warehousing, so as to achieve the purpose of continuously increasing data resources.
First of all, from cognitive psychology, in daily life, people tend to refer to familiar, tangible, and specific experiences to understand that experience and understanding are intangible, difficult to define thinking patterns, construction and understanding. The concept of the relationship between the conceptual domain and the conceptual structure of the target domain is based on the structural relationship between different concepts and the information-based thinking model. This information-based way of thinking is based on the connection between familiar things and unfamiliar concepts, and interface design based on cognitive theory. Secondly, the information society's demand for emotions, after the 1990s, the society continues to develop into the trend of informationization, only material needs can not satisfy consumers, users' subjective feelings are constantly increasing, human-computer interaction is increasing, and emotional needs are more intense. The design begins to pay attention to people's emotional factors. In order to find more accurate, accurate and obvious semantics, the user can actively interact with the product as much as possible, experience, trust, etc.; use the symbol carrier to display the deep meaning of the interface. The information design of the interface itself can give the user some information or meaning, guide the user how to operate the function, the friendly design can help the user to recognize and manipulate, the designer can convey the attribute link of the function through appropriate graphics, making us more familiar The way to present abstract functional meaning. It adopts the shape of positioning, and the shape adopts hexagonal shape to more accurately customize the customer. The elements of the art paint board are used to indicate that the combination of sensibility and rationality can help the designer to Good personalized product design. Using a blue-biased color with the logo of the English comment below, the background color uses a darker notebook as the background, which is a more popular style and conforms to the user's aesthetic. We can follow the user participation in the design principle, because the user characteristics of the participating testers are different, and it has to be determined according to the actual situation. Due to the nature of the software system itself, we have no way to determine the type of user participation. The overall style of the interface uses modern popular elements as the core to develop the design. The interface graphic uses a simple thin outline to outline the icon, fill in the warm red color to highlight the directivity of each option, plus the effect of yang and yin to increase command and navigation. Indicates operability.

\section{CONCLUSION}

Personalized product design is a hot topic, but there are still some shortcomings in the theoretical research of personalized product design, especially the lack of design-related modeling elements. The focus of this paper is to propose a kind of user characteristics based on set theory. The algorithm that derives the type of product modeling elements and its importance degree forms a process of design method, grasps the direction of product design, and has guiding significance for the designer's work. 


\section{ACKNOWLEDGMENT}

Guang Dong University Of Science \&Technology Hospital level project: <Dongguan intangible cultural heritage "thousand corner lamp" skills into the visual communication design professional teaching practice research>.Project number: CQ2016042.

Guang Dong University Of Science \&Technology Hospital level project: $<$ Research on the current situation and trend of visual communication design in Dongguan>. Project number: GKY-2017KYQN-20.

Guang Dong University Of Science \&Technology Hospital level project: $<$ Research on the value and development trend of Dongguan soft decoration design industry>. Project number: GKY-2017KYQN-21.

\section{REFERENCES}

[1] Zhao Qiufangs. Sensible Engineering and Its Research Status in Japan[J]. Art and Design, 12013,04:33-34

[2] Liu Sha. Research on the Color Sensual Experience of Agricultural Machinery Products Based on SD Scale [J]. Journal of Agricultural Mechanization Research, 2010,(4):50-57.

[3] Han Ting, Hu Jieming, Sun Shouqian. Research on Sensory Imagery of Fabric Materials Based on User Perception [J]. Journal of Textile Research, 2012, 33(5): 140-144.

[4] Liang Liang, Zhou Jun. Optimization of Personalization Degree in MC Mode[J].Chinese Management Science,2012,10(6):59-65.

[5] Jing Zhang. A method for using solid modeling CAD software to create an implant library for the fabrication of a custom abutment[J]. The Journal of Prosthetic Dentistry, 2016. 